\title{
Constrained Coding Techniques for the Suppression of Intrachannel Nonlinear Effects in High-Speed Optical Transmission
}

\author{
Ivan B. Djordjevic, Member, IEEE, and Bane Vasic, Senior Member, IEEE
}

\begin{abstract}
A problem of suppression of intrachannel nonlinearities through the use of constrained coding is considered. Three different techniques are proposed and compared with respect to their efficiency, namely 1) constrained coding; 2) combined constrained and error control coding; and 3) deliberate error insertion. Significant $Q$-factor improvement up to $16 \mathrm{~dB}$ depending on code rate and number of spans is demonstrated. A combined constrained-iterative forward error correction (FEC) scheme can operate in the presence of strong intrachannel nonlinearities when even advanced FEC schemes would be overwhelmed with errors. It provides a coding gain of $12.1 \mathrm{~dB}$ at a bit error rate (BER) of $10^{-9}$. Deliberate error insertion is an efficient approach to balance the encoder complexity and the achievable coding gain.
\end{abstract}

Index Terms-Constrained coding, intrachannel crossphase modulation, intrachannel four-wave mixing, optical communications.

\section{INTRODUCTION}

A S OPPOSED to $10-\mathrm{Gb} / \mathrm{s}$ optical transmission systems where nonlinear effects are due to interchannel interactions in high-speed systems (at $40 \mathrm{~Gb} / \mathrm{s}$ and above), the major nonlinear penalties are due to intrachannel interactions, such as intrachannel four-wave mixing (IFWM) and intrachannel crossphase modulation (IXPM) [1]-[7], [16], [17] (these effects are briefly introduced in Section II). The strongest interaction occurs in the regime where the pulses partially overlap, and in order to keep the IXPM low, the dispersion map may be designed so that pulses are spread over many bit periods (the regime is known as pseudolinear transmission). However, in the pseudolinear regime, the second intrachannel nonlinear effect, IFWM, is also present [1]-[4], resulting in energy transfer among pulses within the same wavelength channel. Approaches for dealing with intrachannel nolinearities may be classified into two broad categories, namely 1) modulation formats [2]-[4] and 2) constrained codes [5]-[7]. Both approaches may be combined with a design of proper dispersion map (which is

Manuscript received April 18, 2005; revised August 29, 2005. This work was supported by the National Science Foundation (NSF) under Grants ITR 0325979 and CCR 0208597.

I. B. Djordjevic is with the Department of Electrical and Computer Engineering, University of Arizona, Tucson, AZ 85721 USA, on leave from the Faculty of Computing, Engineering and Mathematical Sciences, University of the West of England, Bristol BS16 1QY, U.K. (e-mail: ivan@ece.arizona.edu).

B. Vasic is with the Department of Electrical and Computer Engineering, University of Arizona, Tucson, AZ 85721 USA (e-mail: vasic@ece. arizona.edu).

Digital Object Identifier 10.1109/JLT.2005.860139 also an important problem but is beyond the scope of this paper). Since the IFWM interaction is a phase-sensitive effect, the aim of the first approach is to remove the phase shortterm coherence of the pulses emitted by the optical transmitter. The role of a constrained code is to avoid waveforms in the transmitted signal that will most likely be detected incorrectly. This approach has been carefully examined by the authors in a series of recent articles, e.g., [5] and [6], and significant performance improvements have been demonstrated for a variety of constraints and dispersion maps.

In this paper, we study three different approaches for suppression of intrachannel nonlinearities, namely 1) the use of constrained encoding; 2) combined constrained and error control coding; and 3) deliberate error insertion.

The constrained encoding (considered in Section III) is based on the following three principles: 1) the most troublesome sequences are identified and forbidden; 2) the zero symbol in the so-called "resonant positions" is converted into the one symbol; and 3) the different contributions to a ghost pulse creation cancel each other in resonant positions. It also provides flexibility in selecting the code rate and the nonlinear effect suppression factor.

Even though the constrained decoding described above is conceptually simple (it is based on the sliding-window decoder [8], [9]), it decouples the channel and the error correction decoder, because it operates on hard bits and does not produce soft information, which is necessary for iterative [low-density parity check (LDPC)/turbo] decoding. An alternative scheme that circumvents the above problem is known as reverse concatenation, and it has been recently adapted for soft detectors. In this scheme, the Bahl, Cocke, Jelinek, and Raviv (BCJR) algorithm [12] is performed on a trellis corresponding to the constrained graph (for more details, the reader is referred to [13]). The reverse concatenation scheme suitable for suppression of the intrachannel nonlinear effects is described in Section IV. The combined constrained and error control coding can operate in the presence of strong intrachannel nonlinearities when even advanced forward error correction (FEC) schemes would be overwhelmed with errors.

The motivation for the deliberate error insertion technique is the fact that the design of a good high rate constrained code is a challenging problem when the desired constraint is complicated, because the encoder and decoder might be unacceptably complex for high-speed applications, and large decoding window size may result into unacceptable error propagation [18] 
(the effect of error propagation is explained in detail in [9]). The number of error caused by error propagation due to increased decoding window size may result in exceeding error correction capability of the FEC code employed in the standard concatenation scheme [shown in Fig. 10(a) and discussed in Section IV]. Even though combined constrained and error correction scheme described in the previous paragraph provides an excellent coding gain, it requires using encoders and decoders of relatively high complexity. The idea is to trade the redundancy introduced by a constrained code for stronger FEC codes. In other words, only an FEC code is used, while the constraint is imposed on the coded sequence by deliberate error insertion, the technique we recently proposed for magnetic recording systems [18]. This technique is discussed in Section V. The LDPC code is designed to be strong enough to correct both channel errors and errors introduced by the constraint monitoring circuit.

\section{INTRACHANNEL NONLINEAR EFFECTS}

The signal propagation through the transmission medium is modeled by the generalized nonlinear Schrödinger equation (GNLSE) [10] given as

$$
\begin{aligned}
\frac{\partial A}{\partial z}=-\frac{\alpha}{2} A-\frac{i}{2} \beta_{2} \frac{\partial^{2} A}{\partial T^{2}}+ & \frac{\beta_{3}}{6} \frac{\partial^{3} A}{\partial T^{3}} \\
& +i \gamma\left(|A|^{2}-T_{R} \frac{\partial|A|^{2}}{\partial T}\right) A
\end{aligned}
$$

where $z$ is the propagation distance along the fiber, relative time $T=t-z / v_{g}$ gives a frame of reference moving at the group velocity $v_{g}, A(z, T)$ is the complex field amplitude of the pulse, $\alpha$ is the attenuation coefficient of the fiber, $\beta_{2}$ is the group velocity dispersion (GVD) coefficient, $\beta_{3}$ is the second-order $\mathrm{GVD}, \gamma$ is the nonlinearity coefficient giving rise to Kerr effect nonlinearities, namely, self-phase modulation (SPM), crossphase modulation (XPM), and four-wave mixing (FWM), and $T_{R}$ is the Raman coefficient describing the stimulated Raman scattering (SRS).

To study the interactions among the pulses within the channel, the field of a single channel can be decomposed as a sum of fields of individual pulses

$$
A=\sum_{l=1}^{L} A_{l}
$$

where $A_{l}$ represents the $l$ th pulse (out of $L$ ) centered at $t=t_{l}$. GNLSE can be rewritten, after the substitution, as

$$
\begin{aligned}
\sum_{l=1}^{L}\left(\frac{\partial A_{l}}{\partial z}+\frac{\alpha}{2} A_{l}+\frac{i}{2} \beta_{2} \frac{\partial^{2} A_{l}}{\partial T^{2}}\right. & \left.-\frac{\beta_{3}}{6} \frac{\partial^{3} A_{l}}{\partial T^{3}}\right) \\
& =i \gamma \sum_{l, k, m=1}^{L} A_{l} A_{k} A_{m}^{*}
\end{aligned}
$$

(The SRS term was ignored to keep the explanation simpler.) The intrachannel nonlinearities may be identified as follows. The case $l=k=m$ corresponds to $\mathrm{SPM}$, the case $l \neq k \neq m$ or $l=k \neq m$ to IFWM, and the case $l=m \neq k$ or $k=m \neq l$ to IXPM.

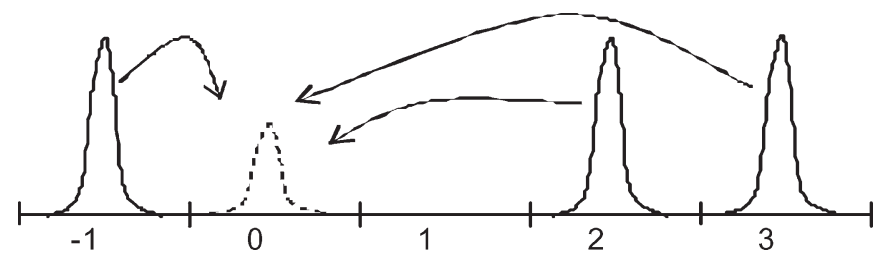

Fig. 1. Pulses at positions 2, 3, and -1 give rise to a ghost pulse at position 0 .

In dispersion-managed systems, pulses undergo periodical widening and compression as they travel during transmission. The strongest interaction is at the point at which the pulses partially overlap, and in order to keep the IXPM low in pseudolinear transmission, the pulses are allowed to spread over many bit periods. However, in the pseudolinear regime, the second intrachannel nonlinear effect, IFWM, is also present [1]-[5]. Dispersed pulses experiencing the nonlinearity see a portion of their filed shifted by a discrete frequency value due to FWM of spectral components within the same wavelength channel. At sufficiently high dispersion, the frequency shift is translated into a discrete time shift located near the middle of a neighboring pulse. This energy transfer among the pulses within the same wavelength channel results in amplitude jitter if energy is transferred to a nonempty bit slot or in ghost pulse creation if the energy is transferred to an empty bit slot, as illustrated in Fig. 1. The pulses at positions $k, l$, and $m$, according to (2), may create a ghost pulse in an empty bit slot located at the $m+k-l$ th position (the resonant condition). Avoiding all such triples is not realistic, since the rate of corresponding constrained code tends to become zero. The challenge is to simultaneously minimize both IXPM and IFWM. For more details on the analysis of intrachannel nonlinear effects, the interested reader is referred to [11].

\section{CONSTRAINED ENCODING}

Various modulation formats have been proposed to tackle the effects of nonlinearities [2], [3]. As the creation of ghost pulses is a phase-sensitive effect, the key idea behind these solutions is to reduce ghost pulses by removing the phase coherence in the emitted pulses in a given neighborhood. In another approach, recently reported in [4], the polarization of adjacent pulses is changed so that they are orthogonal to each other.

The role of a constrained code, however, is to impose certain constraints on a transmitted sequence in order to avoid those waveforms that are most likely to be incorrectly received [5], [6]. A constrained encoder translates an arbitrary user bit stream into a bit stream that satisfies the constraints of the channel. Three different constrained approaches successful in the suppression of intrachannel nonlinearities are proposed in the remainder of the section.

\section{A. Constraint A}

Recently, we identified the resonant sequences [5] like "1101," "1011," and "11011" as the most troublesome. The directed graph of the constrained system avoiding those sequences is shown in Fig. 2. Valid sequences can be obtained by reading off the edge labels while making transitions from 


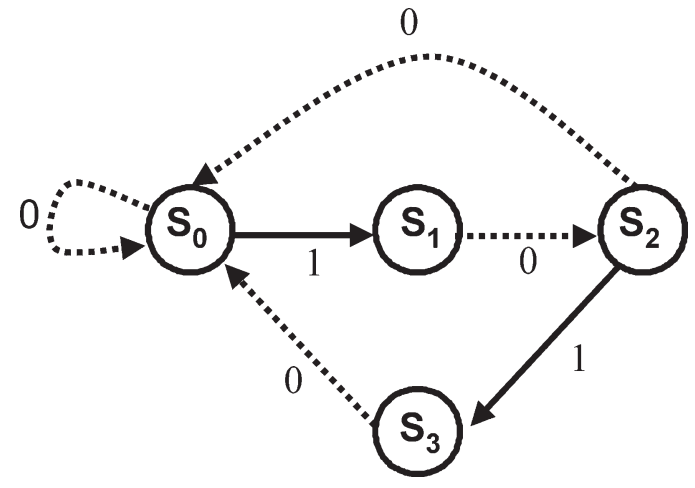

Fig. 2. Directed graph a constraint avoiding the resonance patterns "1101," "1011," and "11011."

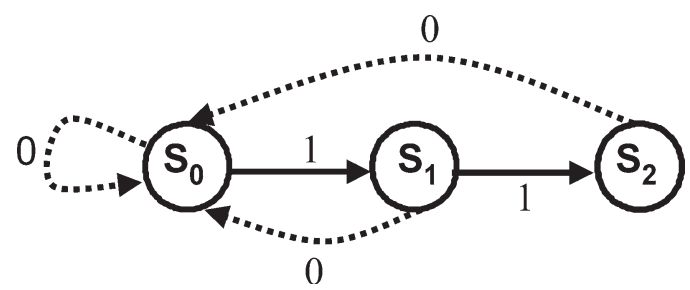

Fig. 3. Directed graph of $(0,2)$ run-length-limited (RLL) constraint (the number of successive $1 \mathrm{~s}$ is constrained between 0 and 2).

TABLE I

DiRECTED GRAPH FOR THE 4/5 RATE CODE

\begin{tabular}{cccc}
\hline Input & Codeword & Input & Codeword \\
\hline 0000 & 00000 & 1000 & 00110 \\
0001 & 00001 & 1001 & 01000 \\
0010 & 00101 & 1010 & 01010 \\
0011 & 01001 & 1011 & 01100 \\
0100 & 10001 & 1100 & 10000 \\
0101 & 10101 & 1101 & 10010 \\
0110 & 00010 & 1110 & 10100 \\
0111 & 00100 & 1111 & 10110 \\
\hline
\end{tabular}

one state to another according to the orientation of the edges. The largest code rate permitted by this constraint (capacity) is 0.6942. Since the most troublesome sequences are identified and forbidden, this constraint will result in excellent $Q$-factor improvement; however, the code rate is unacceptably low for high-speed transmission.

\section{B. Constraint B}

Consider a sequence of length $L, c_{i} c_{i+1} \ldots c_{i+L-1}$, satisfying the following constraint: If for $k, l, m \in[i, i+L)$ and $k+l-m \in[i, i+L)$ ( $k$ and $l$ not necessarily distinct), $c_{k}=c_{l}=c_{m}=1$, then $c_{k+l-m} \neq 0$. If the above sequence is transmitted, then no ghost pulse will be created at any position (positions $\{0,1, \ldots, L-1\}$ ). However, the amplitude jitter may be caused through the IFWM process described by (2). For $L=4$, the constraint has the capacity (the largest possible code rate) of 0.8791 and may be described by a directed graph in Fig. 3. The high-speed applications require the simplest possible realization, and a simple block code of rate $4 / 5$ is given in Table I. The code is designed using the approach described in $[8$, Ch. 5] and has the efficiency (code rate/capacity) of $91 \%$.

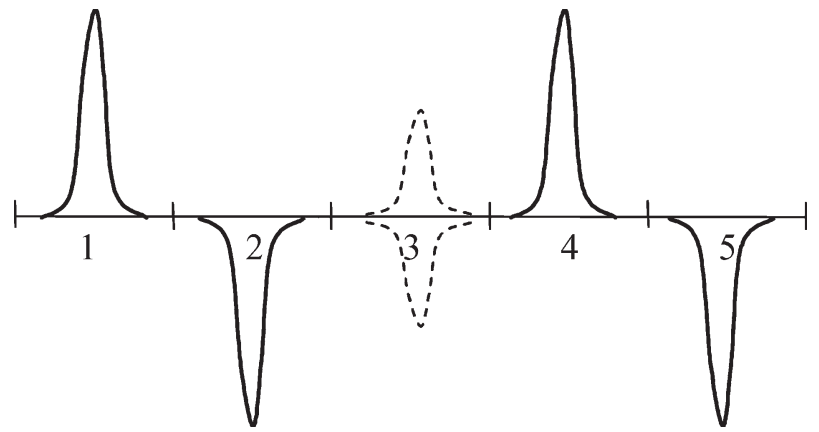

Fig. 4. Pseudoternary cancellation principle. Pulses at positions $1-4-2$ and pulses at positions 2-5-4 creates two ghost pulses of the same amplitude but opposite signs at position 3 .

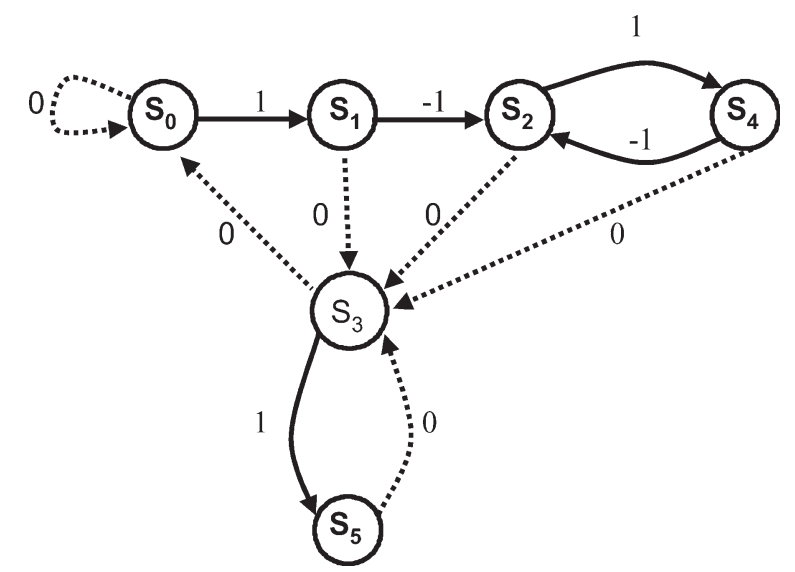

Fig. 5. Pseudoternary code of rate 0.9 .

\section{Constraint C}

The third approach is based on the observation from (2), that the proper choice of initial pulse phases may result in complete cancellation of different ghost-pulse contributors at a zero-bit position in "resonance" (this effect is illustrated in Fig. 4). A pseudoternary constrained code satisfying such property is shown in Fig. 5. The symmetry is imposed by a deliberate addition of a single pulse on the left or right side of "asymmetric" sequence (e.g., the sequence " $1,-1,0,1$ " is translated into " $1,-1,0,1,-1$ "). (Since " 1 " and " -1 " symbols in direct detection are considered as the same symbol, the corresponding constraint is called pseudoternary here.)

\section{Constrained Coding Performance Assessment}

To asses the performance improvement, the $40 \mathrm{~Gb} / \mathrm{s}$ singlechannel long-haul transmission system is simulated. The effects of Kerr nonlinearities (SPM, IXPM, IFWM), SRS, dispersion (GVD, second-order GVD), crosstalk, and intersymbol interference were taken into account. For the light propagation through the fiber, the nonlinear Schrödinger (1) was solved using the split-step Fourier method [10].

The dispersion map, shown in Fig. 6, is composed of $N$ (20-80) spans of length $L=48 \mathrm{~km}$, each span consisting of $2 L / 3 \mathrm{~km}$ of $\mathrm{D}_{+}$fiber followed by $L / 3 \mathrm{~km}$ of $\mathrm{D}_{-}$fiber. The $\mathrm{D}_{+}$ fiber has a dispersion of $20 \mathrm{ps} /(\mathrm{nm} \cdot \mathrm{km})$, a dispersion slope of $0.06 \mathrm{ps} /\left(\mathrm{nm}^{2} \cdot \mathrm{km}\right)$, an effective area equal to $110 \mu \mathrm{m}^{2}$, and 


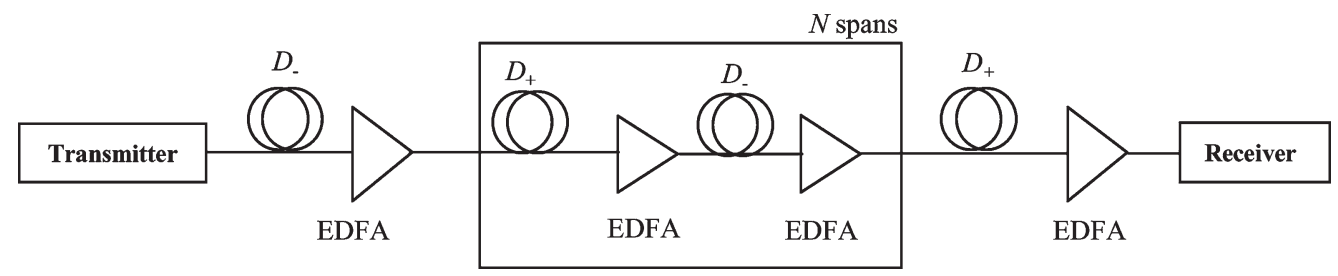

Fig. 6. Dispersion map under consideration.

loss equal to $0.19 \mathrm{~dB} / \mathrm{km}$. The $\mathrm{D}_{-}$fiber has a dispersion of $-40 \mathrm{ps} /(\mathrm{nm} \cdot \mathrm{km})$, a dispersion slope of $-0.12 \mathrm{ps} /\left(\mathrm{nm}^{2} \cdot \mathrm{km}\right)$, an effective area equal to $30 \mu \mathrm{m}^{2}$, and loss equal to $0.25 \mathrm{~dB} / \mathrm{km}$. The nonlinear Kerr coefficient is set to $2.6 \times 10^{-20} \mathrm{~m}^{2} / \mathrm{W}$ in both types of fibers. The precompensation of $-320 \mathrm{ps} / \mathrm{nm}$ and the corresponding postcompensation are also employed. The erbium-doped fiber amplifiers (EDFAs) with a noise figure of $6 \mathrm{~dB}$ are deployed after every fiber section. The simulations were carried out with an average launched power of $0 \mathrm{dBm}$ and a central wavelength of $1552.524 \mathrm{~nm}$. The dispersion map in Fig. 6 is selected in such a way that IXPM is low. However, during transmission over a $D_{+}$fiber, the pulse is spread over up to ten bit periods rather than over tens or hundreds of bit periods, which is typical for pseudolinear transmission. Under such conditions, IFWM is a predominant effect, and the proposed constrained codes are the most efficient.

The results of simulations are given in Figs. 7-9. The proposed constrained codes are compared against the returnto-zero (RZ) modulation format (of duty cycle 33\%). The pattern sequence used in simulations is of length $2^{15}-1$. In Fig. 7, it is evident that the constrained codes are successful in suppressing both IFWM (ghost pulse and amplitude jitter) and IXPM (timing jitter). For the RZ modulation format, the eye diagram after 50 spans is closed, while the eye diagrams for constrained codes are widely open. The ability of constrained codes to substantially reduce the timing jitter due to IXPM is also important in enabling the clock recovery, which would be quite challenging when the eye diagram is completely closed [see Fig. 7(b)].

The $Q$-factor improvement against the number of spans is given in Figs. 8 and 9. The $Q$-factor improvement is defined as

$$
\Delta Q=20 \log \left\lceil\frac{Q_{\text {encoded }}}{Q_{\text {uncoded }}}\right\rceil[\mathrm{dB}] .
$$

Significant $Q$-factor improvement up to $16 \mathrm{~dB}$, depending on code rate and number of spans, is demonstrated. Notice that in the presence of amplified spontaneous emission (ASE) noise, the eye diagrams are more closed due to noise, resulting in reduced $Q$-factor improvement.

\section{COMbined CONSTRAined And ERror CONTROL CODING}

In standard concatenation scheme [Fig. 10(a)], the constrained encoder is placed between an FEC encoder and the channel. However, constrained decoder is usually implemented as a Boolean function applied on a finite number of bits surrounding the bit to be decoded. In other words, the decoder can use only hard decision channel outputs [8], [9] and processes hard bits, therefore, prohibiting soft iterative decoding.

In the reverse concatenation scheme, proposed by Bliss [14], the order of FEC encoder and constrained encoder is inverted. As shown by Fan in [13], such a scheme facilitates the use of soft decoding. The message bits at the output of constrained encoder 1 pass through a systematic LDPC encoder unchanged. Parity check bits in general do not satisfy channel constraint and must be encoded using a separate constrained encoder [constrained encoder 2 in Fig. 10(b)]. Encoder 2, of course, imposes the same constraint as encoder 1 .

Soft constrained decoders 1 and 2 employ the BCJR algorithm [12] performed on the trellis obtained by transforming the state-transition diagram in Fig. 3. The error correction code employed is an LDPC code of length $n$ and dimension (number of message bits) $k$. The message bit reliabilities $L\left(m_{j}\right)$ are calculated in the LDPC decoder using the message-passing algorithm [15]

$$
L\left(m_{j}\right)=\log \left[\frac{p\left(m_{j}=0 \mid \boldsymbol{r}_{\boldsymbol{m}}\right)}{p\left(m_{j}=1 \mid \boldsymbol{r}_{\boldsymbol{m}}\right)}\right]
$$

where $p\left(m_{j}=i \mid \boldsymbol{r}_{m}\right)$ denotes the a posteriori probability of the $j$ th message bit being $i \in\{0,1\}$, given the received message vector $\boldsymbol{r}_{m}=\left(r_{m, 1}, r_{m, 2}, \ldots, r_{m, k}\right)$, where $r_{m, k}$ is the channel output corresponding to the message bit $m_{k}$.

Soft constrained decoder 2 symbol reliabilities $\lambda(\boldsymbol{p})$ are calculated using the BCJR algorithm operating on channel samples corresponding to parity bits $\boldsymbol{p}=\left(p_{1}, p_{2}, \ldots p_{n-k}\right)$. The parity bit reliabilities $L\left(p_{j}\right)$ are calculated from symbol reliabilities as

$$
L\left(p_{j}\right)=\log \frac{\sum_{\boldsymbol{p}: p_{j}=1} \exp [\lambda(\boldsymbol{p})]}{\sum_{\boldsymbol{p}: p_{j}=0} \exp [\lambda(\boldsymbol{p})]} .
$$

The LDPC decoder extrinsic a posteriori reliabilities are fed to the soft constraint decoder 1 (BCJR algorithm) to determine $\lambda(s)$ : the reliability of the source symbols $s$. Finally, source bits $s_{i}$ reliabilities (source bits refer to information bits at the input of the constrained encoder 1, as shown in Fig. 10) are determined by

$$
L\left(s_{j}\right)=\log \frac{\sum_{s: s_{j}=1} \exp [\lambda(s)]}{\sum_{s: s_{j}=0} \exp [\lambda(s)]} .
$$

In calculation of bit reliabilities (4) and (5), the following "maxstar" operator is applied recursively [19]:

$$
\max ^{*}(x, y)=\max (x, y)+\log \left(1+e^{-|x-y|}\right)
$$




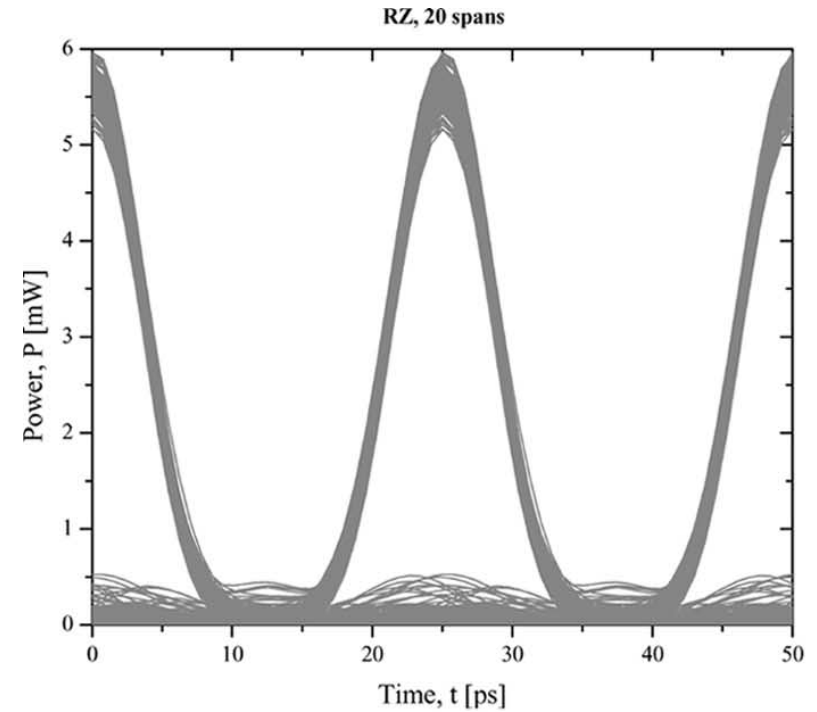

(a)

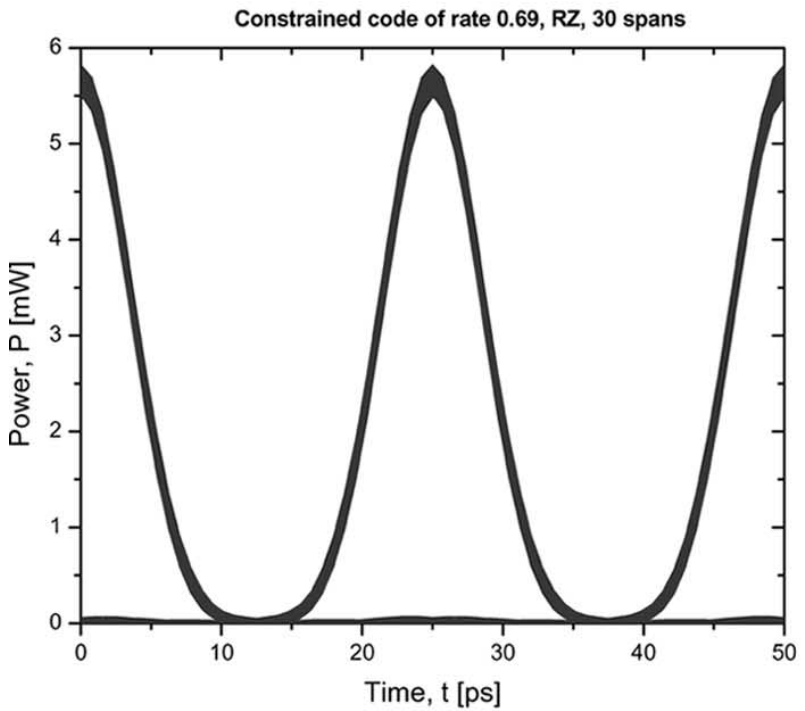

(c)

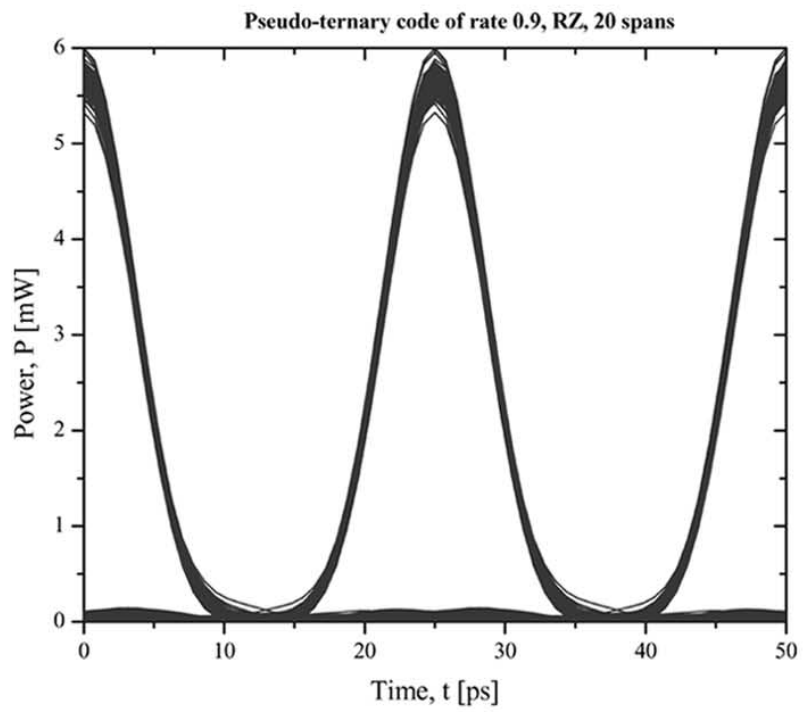

(e)

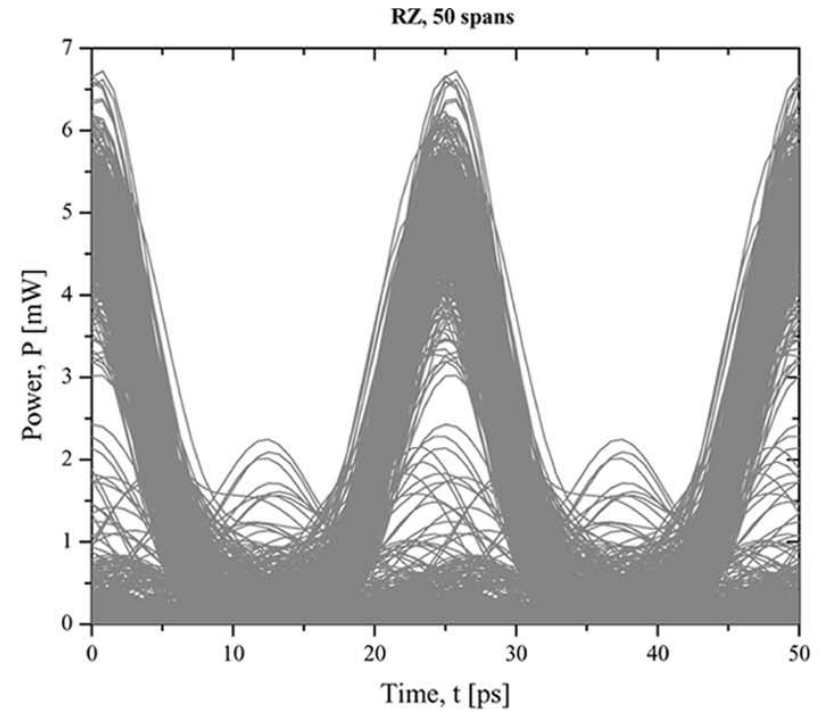

(b)

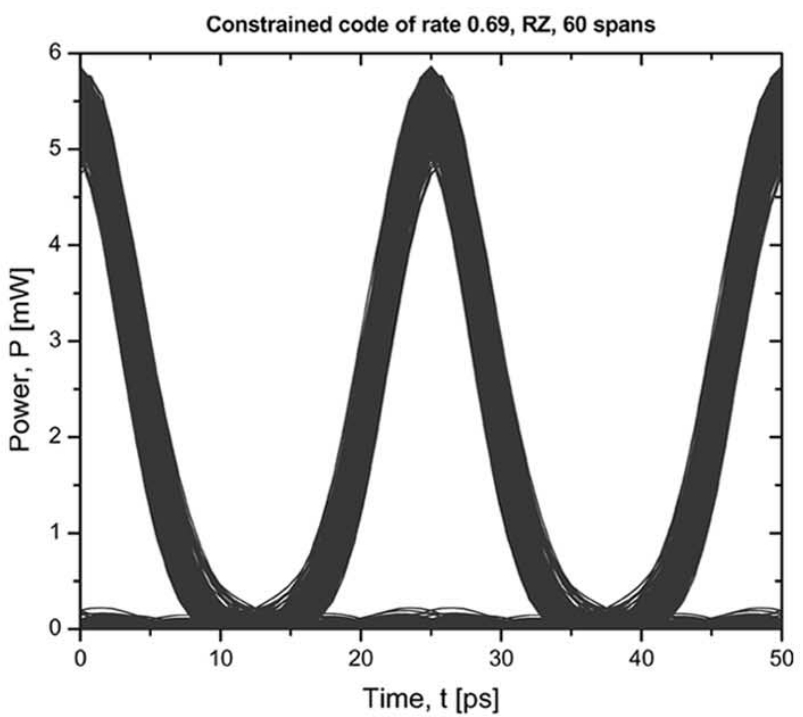

(d)

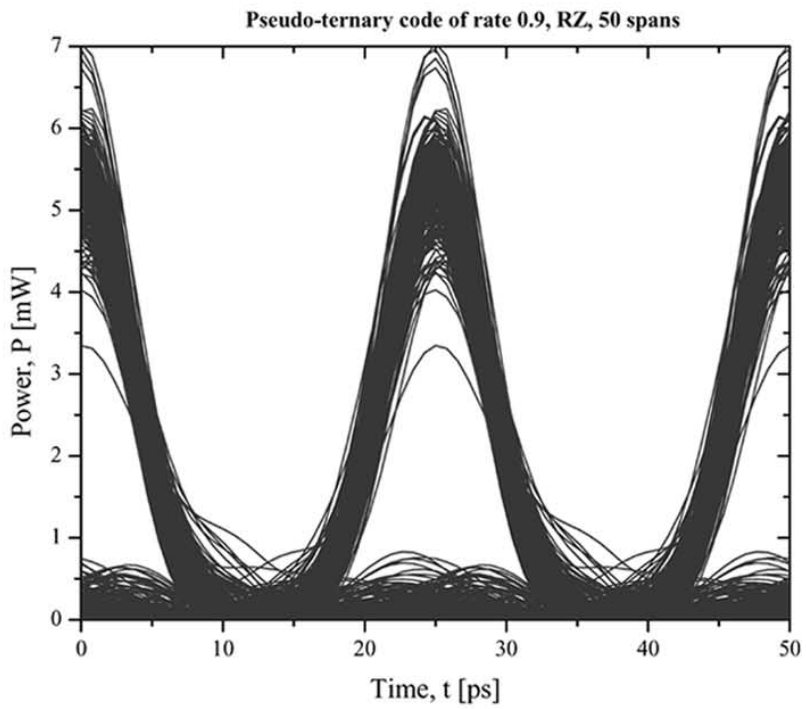

(f)

Fig. 7. Eye diagrams: RZ format, uncoded signal eye diagram after (a) 20 spans and (b) 50 spans. Constrained code of rate 0.69 after (c) 30 spans and (d) 60 spans. Pseudoternary constrained code of rate 0.9 after (e) 20 spans and (f) 50 spans. 


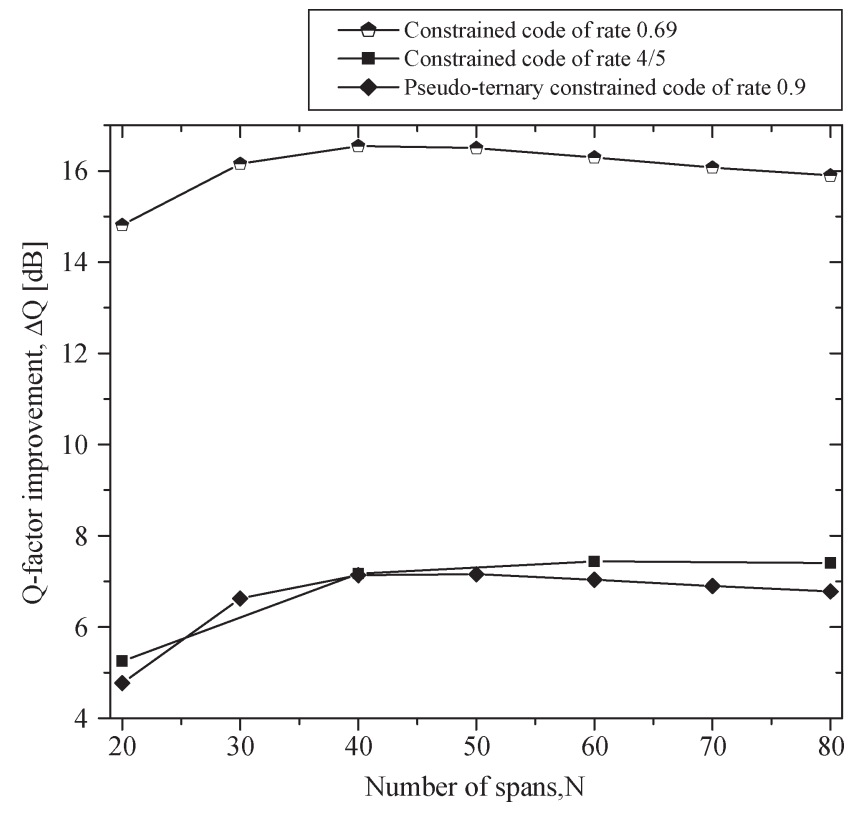

Fig. 8. $Q$-factor improvement over RZ for different number of spans in the absence of ASE noise.

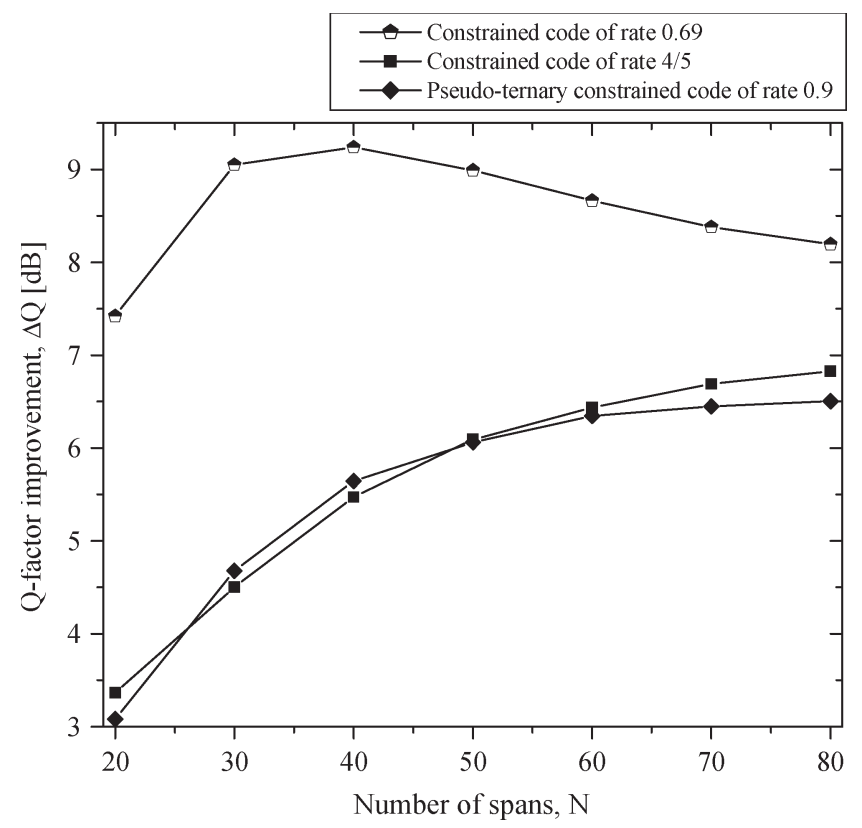

Fig. 9. $Q$-factor improvement over RZ for different number of spans in the presence of ASE noise.

where the max-star operator is defined as $\max ^{*}(x, y)=$ $\log \left(e^{x}+e^{y}\right)$. Notice that the system performance can be further improved by iterating between the LDPC and soft constraint decoders.

The symbol reliabilities $\lambda(\boldsymbol{u})(\boldsymbol{u} \in\{\boldsymbol{p}, \boldsymbol{s}\})$ used to calculate the bit reliabilities in (4) and (5) are determined from the channel samples $\boldsymbol{r}=\left(r_{1}, r_{2}, \ldots\right)$

$$
\lambda(\boldsymbol{u})=\log \frac{P(\boldsymbol{u} \mid \boldsymbol{r})}{P(0 \mid \boldsymbol{r})} .
$$

$P(\boldsymbol{u} \mid \boldsymbol{r})$ is determined using the Bayes' rule

$$
P(\boldsymbol{u} \mid \boldsymbol{r})=\frac{P(\boldsymbol{r} \mid \boldsymbol{u}) P(\boldsymbol{u})}{\sum_{\boldsymbol{v}} P(\boldsymbol{r} \mid \boldsymbol{v}) P(\boldsymbol{v})}
$$

and $P(\boldsymbol{r} \mid \boldsymbol{u})$ is estimated from the channel.

The results of simulation are shown in Fig. 11 for the same dispersion map from Fig. 6, wherein the EDFAs' noise figure is set to $6 \mathrm{~dB}$. After 80 spans, the electrical eye diagram of uncoded signal (shown in Fig. 11) is completely closed, and the input bit error rate (BER) is too high for any FEC scheme to handle (even for the most advanced methods based on turbo or LDPC codes). However, an LDPC code combined with constrained encoder is able to operate error free. The coding gain due to combined constrained and iterative error control coding is $12.1 \mathrm{~dB}$ at a BER of $10^{-9}$. For a BER of $10^{-12}$, the expected coding gain is about $13.6 \mathrm{~dB}$. The resulting coding rate can be determined as a product of code rates of an LDPC code and a constrained code, and it might be unacceptably low, but this is the only alternative to enable transmission in the presence of strong IFWM.

\section{Deliberate Error Insertion}

The key idea behind deliberate error insertion [18], as illustrated in Fig. 12, is to completely remove the constrained encoder and instead impose the constraint by deliberate insertion of errors before the transmission. The redundancy to be used in imposing a given constraint might be used to employ stronger FEC scheme capable of correcting both channel errors and deliberate errors. Constraint monitoring circuit, which replaces the constrained encoder, operates on LDPC-encoded sequence. It monitors if the channel constraint is satisfied; if not, it introduces the smallest possible number of errors so that constraint of the channel is satisfied. The errors introduced by the constraint monitoring circuit are independent of channel conditions and may cause the error floor if the constraint is so strong that the number of introduced errors exceeds the error correction capability of LDPC code. In order to avoid the error floor, we propose the usage of a weaker constraint-a $(0,3)$ constraintas shown in Fig. 13. Moreover, instead of operating on a bit-bybit basis, we suggest the operation on a block-by-block basis. The monitor circuit accepts $K$ bits at a time from an LDPC encoder end checks if the $(0,3)$ constraint is satisfied within the block of length $K$; if not, it flips the minimum number of bits such that the constraint is satisfied. By such a way, we allow for the violation of the constraint between two neighboring blocks, so that the loose $(0,3)$ constraint is obtained; however, LDPC codes of higher code rates may be employed and error floor is avoided, as shown in Fig. 14. At $10^{-9}$, deliberate error insertion provides the coding gain $10.17 \mathrm{~dB}$, while the expected coding gain at a BER of $10^{-12}$ is $11.56 \mathrm{~dB}$.

\section{CONCLUSION}

We introduced and analyzed three novel methods to counter the effects of IFWM and IXPM, namely 1) constrained codes; 


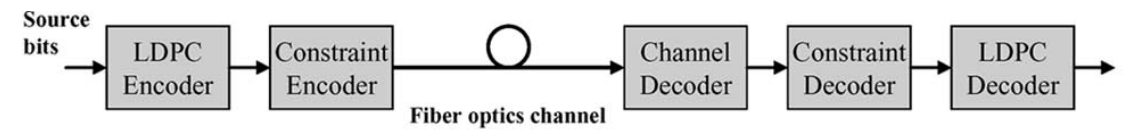

(a)

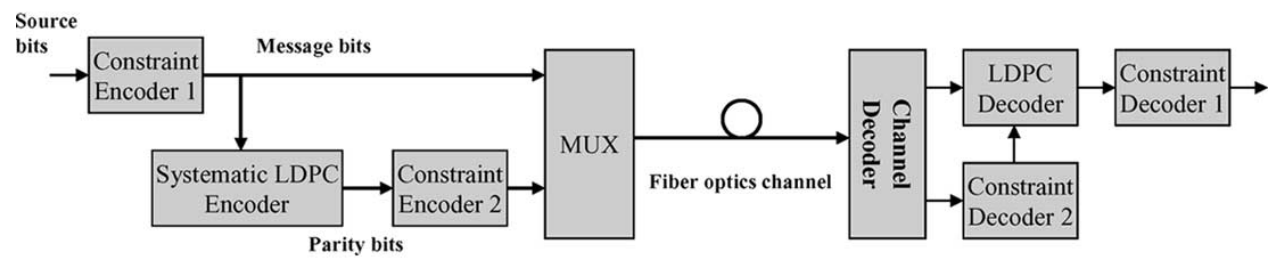

(b)

Fig. 10. (a) Standard concatenation scheme. (b) Reverse concatenation scheme (optical transmitter and receiver parts not shown).

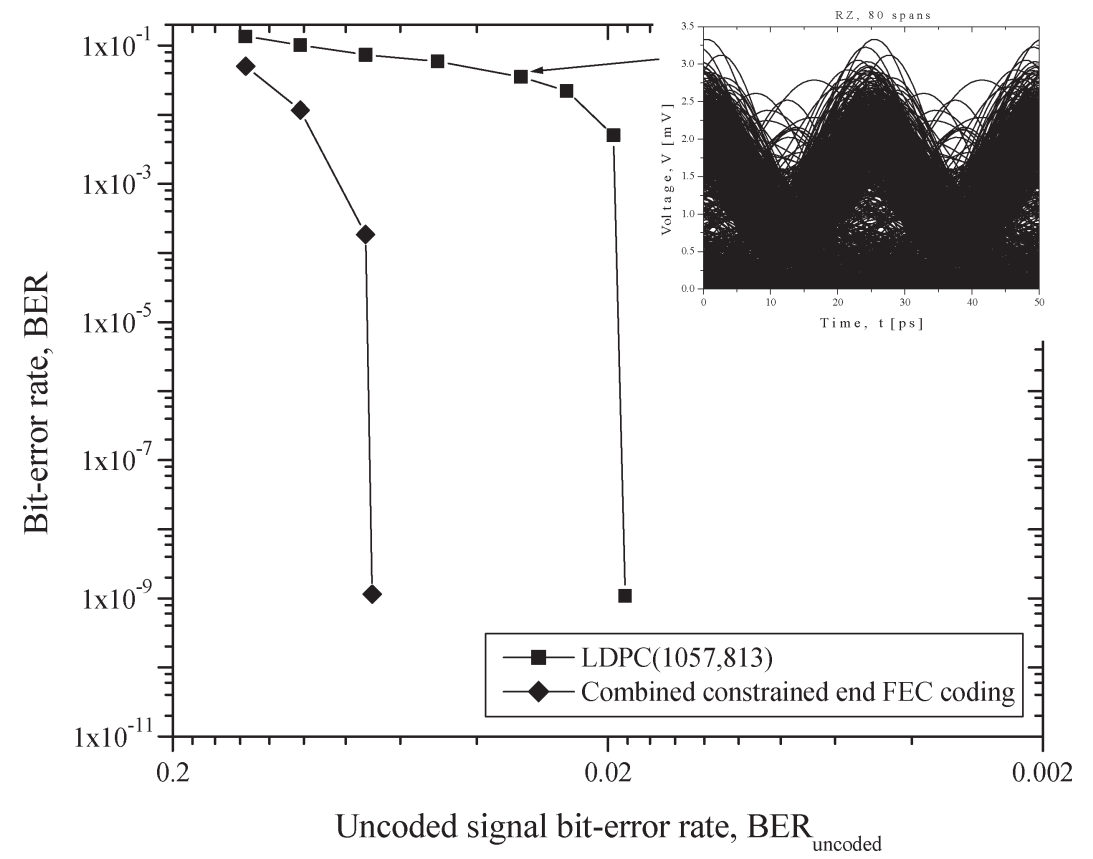

Fig. 11. BER performance of combined constrained and LDPC FEC scheme at $40 \mathrm{~Gb} / \mathrm{s}$. LDPC code belongs to the class of projective geometry codes (our article [20]).

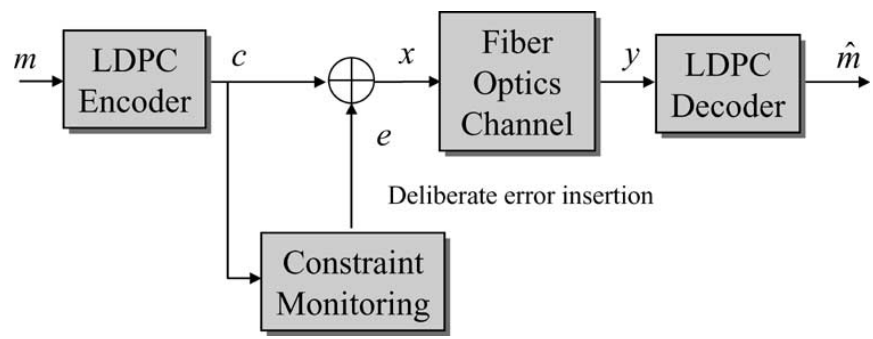

Fig. 12. Deliberate error insertion principle (optical transmitter and receiver parts not shown).

2) combined constrained and error control coding; and 3) deliberate error insertion.

Significant $Q$-factor improvement up to $16 \mathrm{~dB}$, depending on code rate and number of spans, is demonstrated. Combined constrained and error control coding is able to operate in the regime of strong intrachannel nonlinearities when even the most advanced FEC schemes are not able to operate. At $10^{-9}$, it

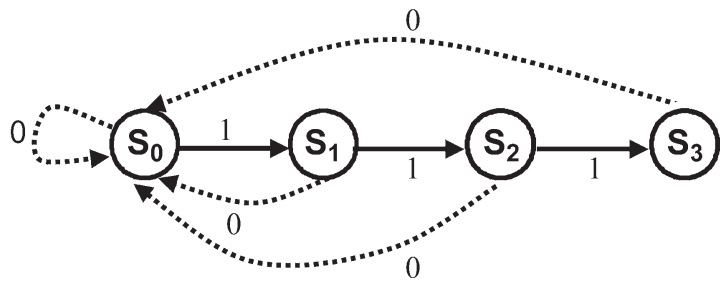

Fig. 13. Directed graph of $(0,3)$ RLL constraint.

provides the coding gain of $12.1 \mathrm{~dB}$, while the expected coding gain at a BER of $10^{-12}$ is $13.6 \mathrm{~dB}$. Deliberate error insertion is the simplest approach to suppress the intrachannel nonlinearities: The constrained encoder is removed completely and replaced by the constraint monitoring circuit. In this scheme, the redundancy to be used for both constrained and error control coding is invested in stronger LDPC code capable of correcting both the channel and the deliberate errors. By employing 


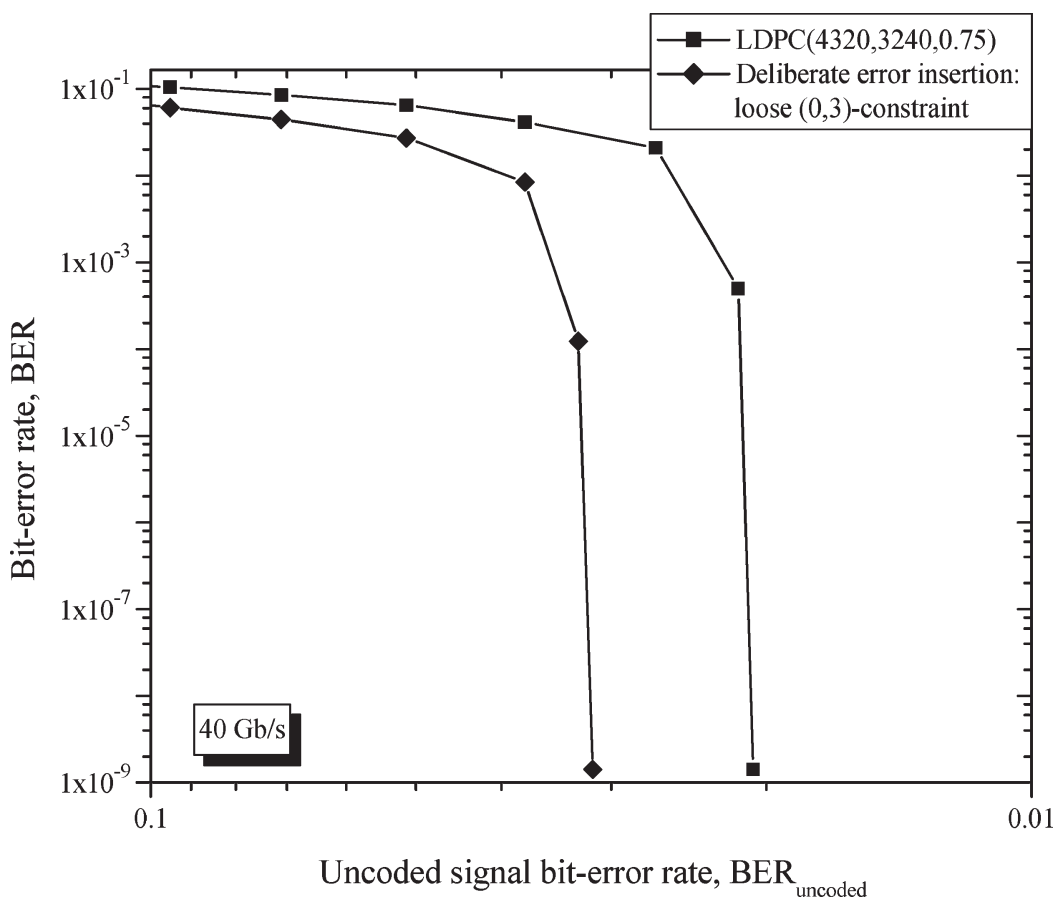

Fig. 14. BER performance of deliberate error insertion scheme for $K=4$. LDPC code belongs to the class of block-circulant codes (our article [21]).

block-circulant $(4320,3240,0.75)$ LDPC code, the deliberate error insertion scheme provides the coding gain of $10.17 \mathrm{~dB}$ at a BER of $10^{-9}$, while the expected coding gain at a BER of $10^{-12}$ is $11.56 \mathrm{~dB}$.

Finally, we would like to point out that the code design is done independently of the design of the dispersion map, and it would be interesting to investigate if a joint optimization leads to further improvements.

\section{ACKNOWLEDGMENT}

The authors thank S. K. Chilappagari for his help while this paper is being written and to the anonymous reviewers whose comments lead to significant improvement of the paper.

\section{REFERENCES}

[1] R. J. Essiambre et al., "Intra-channel cross-phase modulation and fourwave mixing in high-speed TDM systems," Electron. Lett., vol. 35, no. 18, pp. 1576-1578, Sep. 1999.

[2] M. Forzati et al., "Reduction of intrachannel four-wave mixing using the alternate-phase RZ modulation format," Photon. Technol. Lett., vol. 14, no. 9, pp. 1285-1287, Sep. 2002.

[3] X. Liu et al., "Suppression of interchannel four-wave-mixing-induced ghost pulses in high-speed transmissions by phase inversion between adjacent marker blocks," Opt. Lett., vol. 27, no. 13, pp. 1177-1179, Jul. 2002.

[4] C. Xie et al., "Suppression of intrachannel nonlinear effects with alternate-polarization formats," J. Lightw. Technol., vol. 22, no. 3, pp. 806-812, Mar. 2004.

[5] B. Vasic, V. Rao, I. B. Djordjevic, R. Kostuk, and I. Gabitov, "Ghost pulse reduction in $40 \mathrm{~Gb} / \mathrm{s}$ systems using line coding," IEEE Photon. Technol. Lett., vol. 16, no. 7, pp. 1784-1786, Jul. 2004.

[6] I. B. Djordjevic, S. K. Chilappagari, and B. Vasic, "Suppression of intrachannel nonlinear effects using pseudo-ternary constrained codes," J. Lightw. Technol., to be published.

[7] N. Alic and Y. Fainman, "Data-dependent phase coding for suppression of ghost pulses in optical fibers," IEEE Photon. Technol. Lett., vol. 16, no. 4, pp. 1212-1214, Apr. 2004.
[8] D. Lind and B. Marcus, Symbolic Dynamics and Coding. Cambridge, UK: Cambridge Univ. Press, 1995.

[9] B. Marcus, P. Siegel, and R. Roth, "An introduction to coding for constrained systems," in Handbook of Coding Theory, W. C. Huffman and V. Pless, Eds. New York: Elsevier, 1998.

[10] G. P. Agrawal, Nonlinear Fiber Optics. San Diego, CA: Academic, 2001.

[11] A. Mecozzi, C. B. Clausen, and M. Shtaif, "Analysis of intrachannel nonlinear effects in highly dispersed optical pulse transmission," IEEE Photon. Technol. Lett., vol. 12, no. 4, pp. 392-394, Apr. 2000.

[12] L. R. Bahl, J. Cocke, F. Jelinek, and J. Raviv, "Optimal decoding of linear codes for minimizing symbol error rate," IEEE Trans. Inf. Theory, vol. IT-20, no. 2, pp. 284-287, Mar. 1974.

[13] J. L. Fan and J. M. Cioffi, "Constrained coding techniques for soft iterative decoders," in Proc. Global Telecommunications (GLOBECOM), Rio de Janeireo, Brazil, 1999, pp. 723-727.

[14] W. G. Bliss, "Circuitry for performing error correction calculations on baseband encoded data to eliminate error propagation," IBM Techn. Discl. Bul., vol. 23, pp. 4633-4634, 1981.

[15] S. Sankaranarayanan and B. Vasic, "Message-passing algorithm," in Coding and Signal Processing for Magnetic Recording Systems, B. Vasic, Ed. Boca Raton, FL: CRC, 2005, pp. 10-1-10-18.

[16] V. Rao, I. B. Djordjevic, and B. Vasic, "Application of modulation codes to ghost pulse suppression," in Proc. IEEE/LEOS Workshop Advanced Modulation Formats, Rio Grande, PR, 2004, pp. 29-30.

[17] I. B. Djordjevic and B. Vasic, "A ternary modulation code for suppression of intrachannel nonlinear effects in high-speed optical transmission," presented at the Optical Fiber Communication Conference and Exposition and The National Fiber Optic Engineers Conference on CD-ROM (Optical Society of America, Washington, DC), Anaheim, CA, 2005, Paper OThW2.

[18] B. Vasic and K. Pedagani, "Run-length-limited low-density parity-check codes based on deliberate error insertion," IEEE Trans. Magn., vol. 40, no. 3, pp. 1738-1743, May 2004.

[19] W. E. Ryan, "Concatenated convolutional codes and iterative decoding," in Wiley Encyclopedia in Telecommunications, J. G. Proakis, Ed. New York: Wiley, 2003.

[20] I. B. Djordjevic, S. Sankaranarayanan, and B. Vasic, "Projective plane iteratively decodable block codes for WDM high-speed long-haul transmission systems," J. Lightw. Technol., vol. 22, no. 3, pp. 695-702, Mar. 2004.

[21] O. Milenkovic, I. B. Djordjevic, and B. Vasic, "Block-circulant low-density parity-check codes for optical communication systems," IEEE/LEOS J. Sel. Topics Quantum Electron., vol. 10, no. 2, pp. 294-299, Mar./Apr. 2004. 


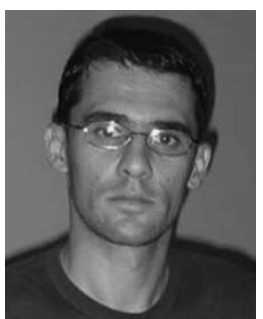

Ivan B. Djordjevic (M'04) received the Dipl.-Ing., M.Sc., and Ph.D. degrees from the University of Nis, Nis, Serbia, in 1994, 1997, and 1999, respectively, all in electrical engineering.

From 1994 to 1996, he was with the University of Nis; and from 1996 to 2000 , he was with the State Telecommunications Company (Serbia Telecom), District Office for Networks, Nis. He was involved in digital transmission systems commissioning and acceptance, design, maintenance, installation, and connection. From 2000 to 2001, he was with the National Technical University of Athens, Greece, and TyCom U.S. Inc. (now TyCo Telecommunications), Eatontown, NJ. He was involved in modeling and simulation of wavelength division multiplexing (WDM) systems and networks. During 2002 and 2003, he was with the University of Arizona, Tucson, University of Bristol, Bristol, U.K., and University of the West of England, Bristol, working on forward error correction and iterative decoding for optical transmission, optical code division multiple access (CDMA), high-speed transmission, and optical switches. He is now with the University of Arizona, on leave from the University of the West of England. He is the author of more than 100 international journal articles and international conference papers. His research interests include dense WDM (DWDM) fiber-optic communication systems and networks, error control coding, CDMA, optical packet switching, coherent communications, free-space optics, statistical communication theory, and satellite communications.

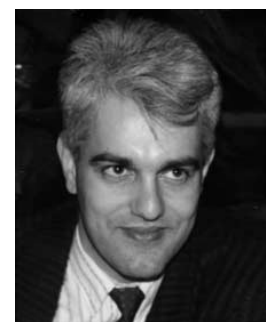

Bane Vasic (S'92-M'93-SM'02) received the B.Sc., M.Sc., and Ph.D. degrees from the University of Nis, Nis, Serbia, in 1990, 1991, and 1994, respectively, all in electrical engineering.

From 1996 to 1997, he worked as a Visiting Scientist at the Rochester Institute of Technology and Kodak Research, Rochester, NY, where he was involved in research in optical storage channels. From 1998 to 2000, he was with Lucent Technologies, Bell Laboratories. He was involved in research coding schemes and architectures for high-speed applications. He was involved in research in iterative decoding and low-density parity check codes, as well as development of codes and detectors implemented in Lucent (now Agere) chips. Presently, he is a Faculty Member of the Electrical and Computer Engineering Department, University of Arizona, Tucson. His research interests include coding theory, information theory, communication theory, and digital communications and recording.

Dr. Vasic is a member of the Editorial Board of the IEEE TRANSACTIONS on Magnetics. He served as a Technical Program Chair of the IEEE Communication Theory Workshop, 2003, and as a Co-Organizer of the Center for Discrete Mathematics and Theoretical Computer Science (DIMACS) Workshops on Optical/Magnetic Recording and Optical Transmission, and Theoretical Advances in Information Recording, 2004. He is a Co-Organizer of the Communication Theory Symposium within the International Conference on Communications (ICC 2006). 